\title{
A Document-Based Electronic Health Record System Controlling the Release of Clinical Documents Using an Access Control List File Based on the HL7 Clinical Document Architecture Header
}

\author{
Toshihiro Takeda*, Akito Nakagawa, Shirou Manabe, Akiko Sakai, Kanayo Ueda and Yasushi Matsumura \\ Department of Medical Informatics, Graduate School of Medicine, Faculty of Medicine, Osaka University, Osaka, \\ Japan
}

\begin{abstract}
Background: Electronic health record (EHR) systems are necessary for sharing medical information between care delivery organizations (CDOs). While many standardized data can be shared, it is still difficult to share nonstandardized clinical data. Furthermore, there remains the problem that the EHR system cannot finely control the disclosure status. We have been operating a documentbased electronic medical record system called the Document Archiving and Communication System (DACS) in which printed images of all medical records are stored in PDF format.
\end{abstract}

Objectives: To develop a document-based EHR system that can disclose selected clinical documents in PDF format.

Methods: With a document-based EHR system, any documents stored in the DACS can be opened to a designated CDO. These open documents can be selected by the service $\mathrm{CDO}$ staff. In order to manage the disclosure status, an access control list (ACL) file was designed based on the HL7 clinical document architecture header. The PDF file and its $A C L$ file were transiently stored in the Japanese standard repository, SS-MIX2. The doctor of the designated CDO was then able to access the documents according to the information in the ACL file.

Results: From March 2017 to September 2017, 308 documents of 20 patients were disclosed to $3 \mathrm{CDO}$ s using this document-based EHR system. These documents included examination reports, agreement forms concerning the EHR, progress notes, summaries and surgical reports generated from five different systems.

Conclusions: The document-based EHR system was able to reveal clinical documents from the EMR to medical staff under a controlled disclosure environment..

\section{Keywords}

Electronic health record; Electronic medical record; Document-based Management of Medical Information; Clinical Document Architecture

\section{Correspondence to:}

Toshihiro Takeda

Department of Medical Informatics,

Graduate School of Medicine,

Osaka University, Osaka, Japan.

E-mail: ttakeda@hp-info.med.osaka-u.ac.jp
EJBI 2018; 14(4):29-38

Received: June 25, 2018

Accepted: August 06, 2018

Published: August 13, 2018

\section{Introduction}

For the smooth continuation of the medical treatment of the patient, it is necessary to share medical information between care delivery organizations (CDOs) using an electronic health record (EHR) system $[1,2]$. The benefits of such a system are improvements in patient safety, medical connectivity and clinical outcomes $[3,4]$. Two architectures have been conceived for the EHR: (1) data are directly entered into the EHR database, or (2) data are primarily entered into the electronic medical record (EMR) database at each CDO and then transferred to the EHR database.

In Japan, the computerized physician's order entry (CPOE) system was implemented at many hospitals in the 1980s and has since been developed into an EMR system. According to the investigation by the Japanese Association of Healthcare Information System Industry (JAHIS) in 2016, $72.9 \%$ of hospitals with more than 400 beds had their own EMR systems [5]. Given this history, Japan should adopt an 
architecture in which the data primarily entered into the EMR database are transferred to the EHR database.

The Standardized Structured Medical Information Exchange (SS-MIX), which is promoted by the Ministry of Health, Labor and Welfare in Japan, is the standard repository for HL7 v2.5 messages [6]. There are two major EHR systems in Japan: ID-Link, manufactured by NEC, and Human Bridge, manufactured by Fujitsu [7]. In each EHR system, the data in the EMR database of each CDO are transferred into the SS-MIX storage and can then be browsed with an EHR viewer. With this EHR system, many standardized data, such as patients' basic information, prescription orders, injection orders, laboratory results and diagnosed diseases, can be shared. However, it is still difficult to share every medical record, especially non-standardized data. These limitations for sharing information associated with the standardized medical information make it difficult to comprehend the medical treatment status of a patient accurately. Furthermore, although sharing information between facilities in the medical and welfare fields is expected to become important in the near future, the records compiled in the welfare field are difficult to standardize.

On the other hand, as the range of shared information expands, new problems will arise. Patients might wish to disclose their clinical information only to the CDOs involved in their medical treatment. Particularly with regard to highly sensitive records, about such as those concerning mental or gynecological illnesses, patients might not wish to disclose these documents even to doctors who have treated them for other diseases. Furthermore, doctors themselves often show resistance to the disclosure of patients' clinical information without their own permission. Doctors may also hesitate to disclose documents written by doctors of other therapeutic departments. To solve this problem, the doctors of service CDO could select which records should be disclosed and to which CDOs the records should be disclosed. The HL7 v3 clinical document architecture (CDA) is a document markup standard that specifies the structure and semantics of „clinical documents“ for the purpose of exchange between CDOs $[8,9]$. The CDA consists of a header and a body. The CDA header includes document information, encounter data, service actors and service targets that can be used to manage information for disclosure.

We developed a document-based EMR system called the Document Archiving and Communication System (DACS) [10]. With the DACS, the print image of all medical records generated by the main system and subsystems in the EMR are stored in PDF format in the DACS database. The nonstandardized data will be able to be shared when the clinical documents are disclosed in PDF format in EHR. In this article, we propose the adoption of a document-based EHR system with the cooperation of the DACS and the existing EHR system where any clinical documents can be disclosed through the DACS database. In order to control the disclosure of the documents, we designed an access control list (ACL) file based on HL7 CDA header. With this system, doctors can disclose any clinical documents according to the designation of the target CDOs.

\section{Methods}

\subsection{Utilizing the existing EHR system, ID-Link}

The existing EHR system is equipped with a secure network (OD-VPN), log-in user management and patient management capabilities (Figure 1). Standardized data, such as prescription orders, injection orders, laboratory results and DICOM pictures, can be browsed with a report viewer and opened using the existing EHR function. Japan has no universal patient identifier available for medical use, so the patient IDs of each CDO must be linked. The existing EHR system is equipped with this function.

\subsection{Managing Document Disclosure Information Using the ACL File}

The ACL contains the data for access control of each clinical document, which is based on the HL7 CDA header. The first group is the document information, such as the document ID, version, class code, title and event date. The second group is the patient information, including the patient ID, name, sex and birthdate. The third group is the originator and generator information, including the facility ID, facility name, originating user ID, originating user name, generator ID and generator name. The fourth group is the facility permitted to view the document, including the facility ID and name.

\subsection{Selecting Target CDOs and Documents for Disclosure Using the DACS Viewer}

The doctors in service CDOs select the target CDOs and the documents which they intend to disclose on the DACS

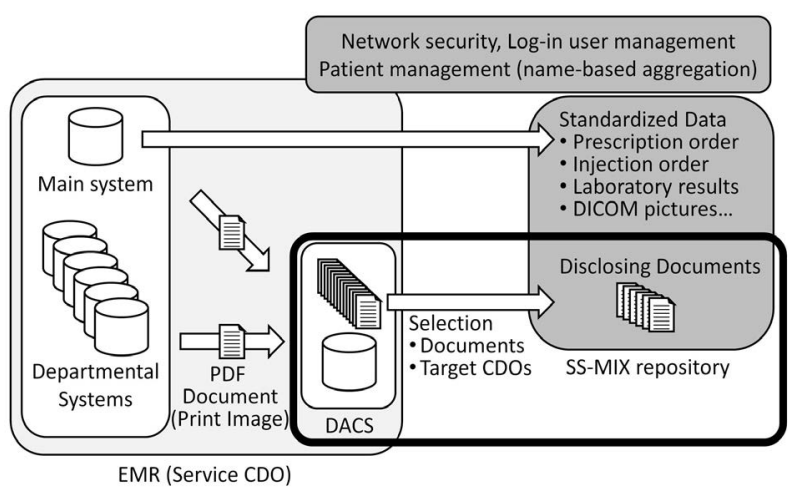

Figure 1: The sharing of data using the existing EHR functions. The data were opened using the existing EHR functions. The functions shown inside the bold frame were implemented in the present study. 
viewer (Figure 2). First, the DACS scrapes the target CDO candidate list from the EHR system (Figure. 2a). Next, the doctors select the target CDOs from the candidate list (Figure 2b). The target CDOs can be determined on a therapeutic department basis. Next, the doctors select the documents for disclosure from the clinical documents stored in the DACS database (Figure 2c). The documents for disclosure can also be determined on a therapeutic department basis. With this information, the DACS generates an ACL file that is transferred into the SS-MIX repository with the selected PDF documents (Figure 2d). The report viewer at the target CDO compiles the list of the documents for disclosure according to the ACL information. A document is then selected by the user at the target CDO, and its PDF can then be browsed.

\subsection{Default Settings for Automatically Disclose the Documents}

Our system enables doctors to set the disclosure status for each clinical document. However, it is difficult for doctors to adjust the settings on documents one at a time. To solve this problem, we designated default settings of the documents for disclosure on a document class basis. Documents with a default disclosure setting can be automatically opened when the doctor selects the target CDO on the DACS viewer. In addition, the documents with a default disclosure setting that are registered to the DACS database after the disclosure operation is completed can also be automatically disclosed to the selected CDOs. The doctors can also delete the disclosure setting of each document on the DACS viewer.

\subsection{Prohibition Settings of the Documents for Disclosure}

Some clinical documents should not be disclosed to the EHR. One is the documents which are judged to prohibit disclosure as the organization, such as documents containing genetic information. Another is the documents that contain sensitive patient information and should be disclosed only by the therapeutic department doctors who created the documents in the first place. Our system sets two prohibition statuses for each document class, as follows: 1) documents whose disclosure is wholly prohibited, and 2) documents that can only be disclosed by the doctor of the department that created the document in the first place.

\subsection{Converting the Local Document Name to a Public Document Name}

The local clinical document name is often freely attached depending on the circumstances of each facility, and in some cases, its use as the public document name in the EHR may be inappropriate. For example, while the document names "Discharge summary (standard format)", "Discharge summary (cardiology format)" and "Heart failure summary" have been used in our EMR system, these document names should be displayed as "Discharge Summary" in EHR. In addition, to ensure efficient browsing, the clinical documents are divided into several categories displayed on the DACS viewer. The local document categories are set so that the clinical documents generated in the hospital are equally divided to some extent. However, the documents disclosed in the EHR are believed to be biased towards certain categories, such as summaries and inspection results (Figure 3). For this reason, in our system, the open documents are divided into other categories that are suitable for EHR.

Therefore, the DACS is equipped with a table for the conversion from a local document code and name to a public document code and name, and the converted public document code and document name are described in the ACL file, which is displayed on the EHR viewer.

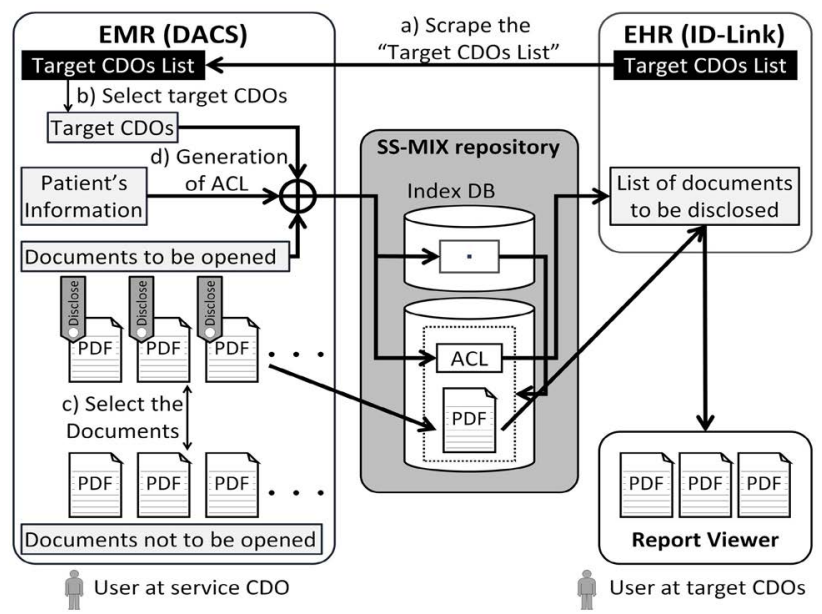

Figure 2: The regulation of document disclosure using the ACL file. The user in the service CDO selects the target CDOs and the documents to be disclosed. The ACL file is then generated based on this information. The users in the target CDOs can browse the documents according to the ACL information.

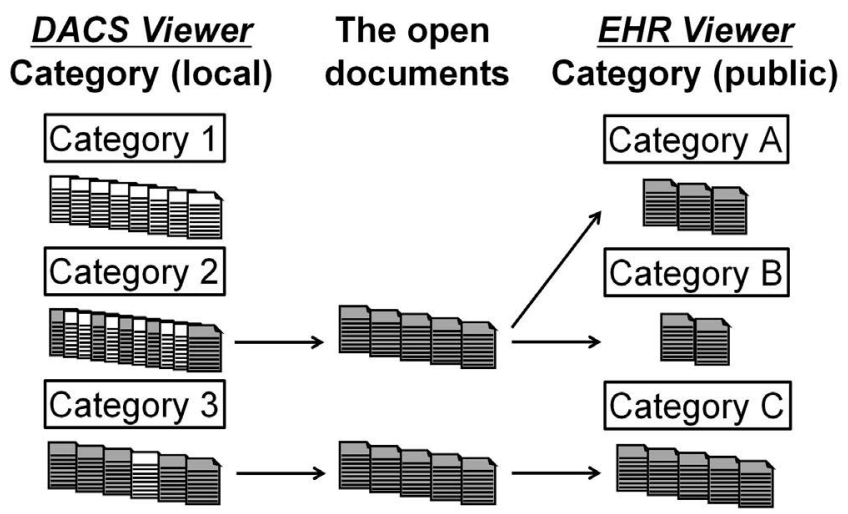

Figure 3: The categories of the clinical documents in the local DACS and EHR viewers. The clinical documents were divided into several categories and displayed in the viewer. Our EHR system defined the original categories, which were independent from the local categories. 


\section{Results}

\subsection{Setting the Disclosure Status on the DACS Viewer}

The disclosure status on the DACS viewer is set as follows: First, doctors of the service CDO push the "disclosure setting" button on the DACS viewer (Figure 4). Next, in the disclosure setting window, the doctors select the target CDOs from the candidate CDO list, set the disclosure period, and push the "disclose" button. The list of default open documents is then displayed so that the doctor can check which documents are going to be disclosed. If the doctors want to disclose the documents with a non-default disclosure setting, they select the documents, check the contents in the documents, and attach the disclosure tag (Figure 5). If the doctors want to revoke the disclosure of the documents, they select the documents and remove the disclosure tag.

\subsection{Default-Open Documents and Setting Prohibitions for Documents for Disclosure}

The medical record management committee of our hospital discussed which documents should be automatically

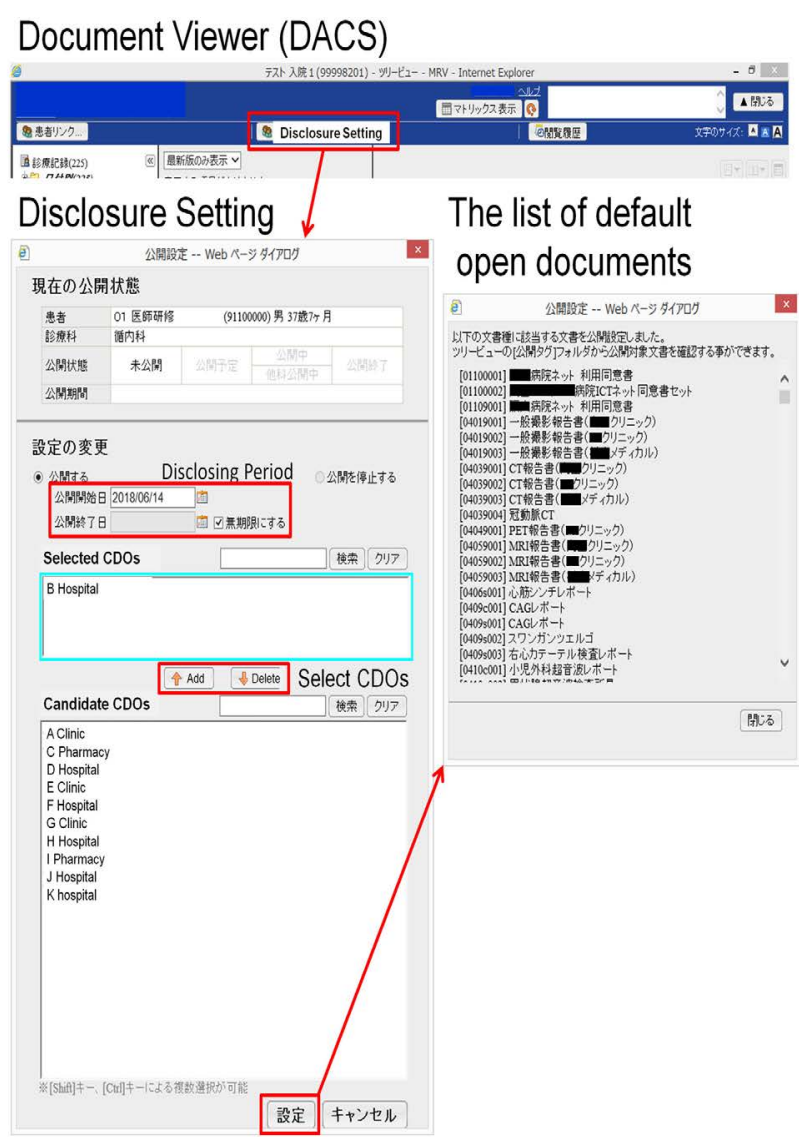

Figure 4: Setting the disclosure status in the DACS viewer. The doctors in the service CDO can select the disclosure settings in the DACS viewer.

\section{Document Viewer (DACS)}

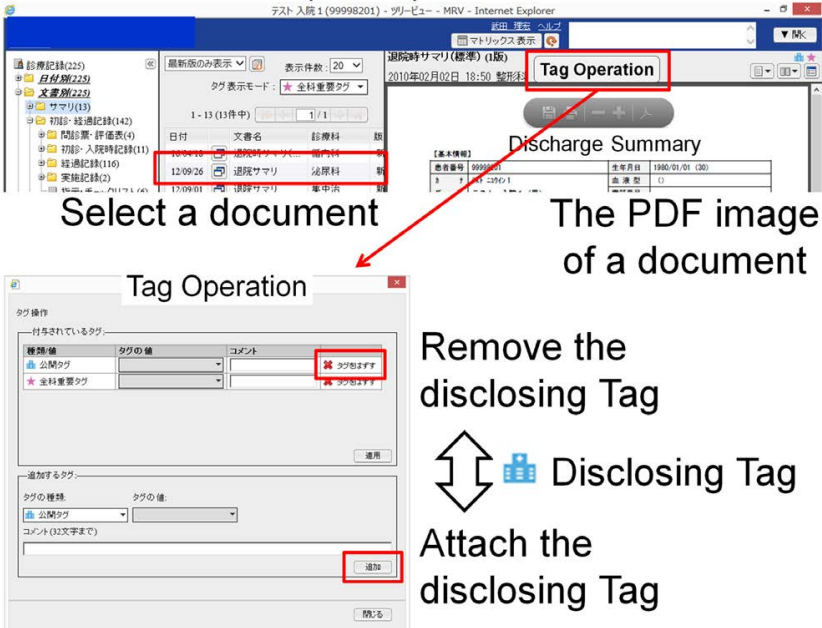

Figure 5: The disclosure tag operation in the DACS viewer. The doctors in the service CDO can set the disclosure status for each clinical document in the DACS viewer.

opened and which documents should be prohibited from being opened (Table 1). The reports of examinations, such as electrocardiogram, radiographic examinations, endoscopic examinations, ultrasound examinations and pathological examinations, as well as the hemodialysis record, radiation exposure record and regional collaboration-related documents were initially set as default-open documents. However, after discussion, the surgical records and discharge summary, which were thought to be key documents for comprehending a patient's medical history, were excluded from the default disclosure status, because they sometimes contain sensitive information for the patients. The disclosure of documents regarding medical costs, cardiopulmonary resuscitation reports and examination reports containing genetic information, was set to be prohibited.

\subsection{Document Viewer of the EHR}

The document viewer of our EHR system was designed to be launched from the existing EHR viewer. The document is displayed in a matrix according to the categories of the document and the event date of the document. The documents categories of the EHR are as follows: 1) Progress note, Flow sheet; 2) Summary; 3) Surgery, Treatment, Delivery reports; 4) Examination reports; 5) Assessment of the patient; 6) Patient creation records; 7) Regional collaboration records and 8) Others. By contrast, the local document categories of our hospital are as follows: 1) Summary, 2) Progress note, Flow sheet; 3) Examination reports; 4) Surgery, Treatment reports; 5) Nursing records; 6) Co-medical records; 7) Consent form, Explanatory document; 8) Document of hospitalization and 9) Introduction letter, Medical certificate. Each document category was established so that the documents are equally displayed. 
Table 1: Clinical document title and their default-open setting used in our document-based EHR system.

\begin{tabular}{|c|c|c|}
\hline Document Class & Document Title & Default-open setting \\
\hline \multirow[t]{4}{*}{ Profile } & Patient profile & \\
\hline & Patient profile for nursing & \\
\hline & Problem list & \\
\hline & Infectious disease information & \\
\hline \multirow[t]{6}{*}{ Summary } & Summary & \\
\hline & Hospitalization summary & \\
\hline & Discharge summary & \\
\hline & Ward round summary & \\
\hline & Outpatient summary & \\
\hline & Nursing summary & \\
\hline \multirow[t]{16}{*}{ Progress Note } & First encounter record & \\
\hline & Progress note & \\
\hline & Nursing record & \\
\hline & Outpatient record (nursing specialty) & \\
\hline & Midwifery record & \\
\hline & Newborn infant record & \\
\hline & Rehabilitation record & \\
\hline & Nutrition management record & \\
\hline & Palliative care record & \\
\hline & Mental healthcare record & \\
\hline & Medical welfare record & \\
\hline & Home medical care record & \\
\hline & Co-medical staff record & \\
\hline & Medical transplantation record & \\
\hline & Gene diagnosis and therapy record & Prohibition \\
\hline & Clinical trial and clinical study record & \\
\hline \multirow[t]{17}{*}{ Medical record } & Nursing care and observation record & \\
\hline & Vital sign sheet & \\
\hline & Risk evaluation sheet & \\
\hline & Medical check list & \\
\hline & Bedsore care record (nursing) & \\
\hline & Nursing operation record & \\
\hline & Medical guidance record & \\
\hline & Growth curve & \\
\hline & Drug-related record & \\
\hline & Contrast media side effect report & $\circ$ \\
\hline & Conference record & \\
\hline & Surgery record & \\
\hline & Nursing record of the surgery & \\
\hline & Anesthesia record & \\
\hline & Invasive procedure record & \\
\hline & Progress note of the examination & \\
\hline & Radiation therapy record & $\circ$ \\
\hline
\end{tabular}


Medical Plan

Communication

Official document

Examination report
Hemodialysis record

Heart-lung machine record

Cardio pulmonary resuscitation record

Prohibition

Interview vote, evaluation list

Document written by the patient

Nutrition management plan

Clinical pass way (in-hospital)

Regional alliances clinical pass way

Introduction letter, referral form (to other facilities)

Introduction letter, referral form (from other facilities)

Medical treatment report (to other facilities)

Medical treatment report (from other facilities)

Request for medical information (to other facilities)

Request for medical information (from other facilities)

Consultation sheet (in-hospital)

Instruction record (from doctor to nurse)

Handover message (from nurse to nurse)

Type 1 style sheet

Medical certificate

Doctor's opinion

Response paper

Consent form

Consent form (Regional alliances)

Public expense related document

Hospitalization related document

Discharge related document

Home medical care related document

Pregnancy related document

Parturient related document

Individual clinical investigation vote

Specific childhood chronic disease document

Medical expenses related document

Prohibition

Medical certification

Prohibition

$\mathrm{X}$-ray (whole body)

X-ray (head)

$\mathrm{X}$-ray (chest)

$\mathrm{X}$-ray (abdomen)

$\mathrm{X}$-ray (limbs)

CT report (whole body)

CT report (head)

CT report (chest)

CT report (abdomen)

CT report (limbs)

MRI report (whole body)

MRI report (head)
○

$\circ$

$\circ$ 
MRI report (chest)

MRI report (abdomen)

MRI report (limbs)

PET report (whole body)

PET report (head)

PET report (chest) 0

PET report (abdomen)

PET report (limbs)

RI report (whole body) 0

$\mathrm{RI}$ report (head)

RI report (chest)

RI report (abdomen) 0

X-ray TV report (whole body)

X-ray TV report (head)

$\mathrm{X}$-ray TV report (chest)

$\mathrm{X}$-ray TV report (abdomen)

X-ray TV report (limbs)

Catheterization report (whole body) 0

Catheterization report (head) 0

Catheterization report (chest) 0

Catheterization report (abdomen) 0

Catheterization report (limbs)

Perioperative X-ray report (whole body) 0

perioperative X-ray report (head) 0

perioperative X-ray report (chest) 0

perioperative X-ray report (abdomen) 0

perioperative X-ray report (limbs) 0

Ultrasonography report (whole body)

Ultrasonography report (head)

Ultrasonography report (chest) 0

Ultrasonography report (abdomen)

Ultrasonography report (limbs)

Gastrointestinal contrasting report 0

Gastric fiber scope report

Colon fiberscope report

Endoscopy report

Electrocardiogram 0

Electroencephalography, magnetoencephalography $\quad$

Physiological examination report 0

Ventilatory function test 0

Bone mineral density measurement $\quad \circ$

Pathology report 0

Laboratory test result 0

Outsourcing examination report 0

Examination result 
Genetic test report

Examination report (specialty field)

Examination report (clinical trial, clinical study)

Examination report (other facilities)
Prohibition

\subsection{Operation Results of Our Document-based EHR System}

The existing EHR system and the document-based EHR system have been in operation since April 2017. As of September 2017, the standardized clinical data of 108 patients were disclosed to 7 facilities using the existing EHR system. Of these 108 patients, non-standardized documents of 20 patients (18.5\%) were disclosed to 3 facilities with our document-based EHR system. Three hundred and eight documents, including examination reports (39 documents), agreement forms on the EHR (18 documents), progress notes (14 documents), introduction letters (8 documents), summaries (4 documents) and surgical reports ( 4 documents), were disclosed. The departmental systems from which the documents were generated were as follows: radiographic examination reporting system (131 documents), physiological examination filing system (51 documents), scan system (43 documents), pathology reporting system (37 documents) and document generation system (32 documents). Among them, 278 documents (90\%) were disclosed automatically with the default setting, and 30 (10\%) were selected to be disclosed by doctors.

\section{Discussion}

In the existing EHR system, the medical information that can be shared is frequently limited to standardized data. However, these standardized data are often insufficient to comprehend a patient's history. The Japanese EMR system usually comprises a main system and a large number of subsystems for special fields, including the document generation system, the pathology reporting system, the radiographic examination reporting system, the ICU system, the ophthalmic system and the obstetrics system, among others, which are thought to be essential for comprehending a patient's history. The data of these systems have not been standardized and require their own special viewing software to browse their contents. While a remote connection through virtual desktop technology enables the target CDOs to browse all of the medical information of a single service CDO, the integration of medical information from several CDOs is difficult. Furthermore, as the range of shared information expands, the regulation of the disclosure of such information will become important, given that sensitive patient information is involved. However, it is difficult to regulate the disclosure information for each patient through remote connection to a local EMR system.
In this study, we developed a document-based EHR system able to disclose any medical documents stored in PDF format in the EMR, including its subsystems. Doctors were able to select target CDOs and documents to be disclosed on a therapeutic department basis under the control of an ACL file based on the HL7 CDA header.

The ACL file defines the event date of the document, not the registration date. The event date is the date on which the patient event described in the document occurred. For example, the event date of the discharge summary is the discharge date, even though the discharge summary is usually registered several days after the discharge date. If the report viewer is displayed in chronological order, it is easier to find documents displayed by the event date than the document registration date.

In the CDA, the HL7/LOINC document ontology, which is an existing document standard, was recommended to be used [11]. However, the ACL file does not use the LOINC code. In the LOINC code, the document name is defined exhaustively, but the documents are not systematically organized. In order to facilitate chronological comparison of the documents from several facilities, it is necessary to organize the thesaurus of document classification and manage the documents with the same granularity. For this reason, the document code described in the ACL file is the code originally defined by our regional alliance. A working group from several hospitals that have adopted document-based EHR systems is presently discussing the standardization of document names.

Cross-Enterprise Document Sharing (XDS) is an interoperability profile that facilitates the registration, distribution and access across CDOs of EHRs $[12,13]$. The repository stores each document, and the registry stores metadata about the documents. The documents can be stored in either or both a PDF or CDA (text-only) format. XDS uses structured EHR standards, such as Care Record Summary, Continuity of Care Record and CDA, to facilitate data exchange. The existing EHR system ID-Link was not designed in compliance with the XDS standard because this system uses a local format to exchange clinical information. However, our document-based EHR system, which stores documents in a PDF format in the repository and the ACL file based on the HL7 CDA header in the registry, complies with the XDS standard.

The selection of the documents to be disclosed is important for several reasons. First, patients want to disclose their sensitive information only to doctors who require 
this information for their medical treatment. The patient is explained what kind of information will be disclosed to the designated $\mathrm{CDO}$ by the doctor. If there is information that the patient does not want to disclose, the patient will be able to ask the doctor to stop disclosing. Second, doctors may sys want to select which documents can be disclosed. Indeed, our medical record management committee decided not to allow the automatic disclosure of the surgical records and discharge summary, documents initially believed to be key for comprehending a patient's medical history. Each doctor can then decide on a patient-by-patient basis whether or not these records should be disclosed. Third, only essential information should be disclosed in the EHR from the perspective of effective medical information collection. At our hospital, hundreds of clinical documents are usually stored in the DACS for patients with a history of hospitalization or a history of outpatient visits for more than one year. Some of these documents must be kept in the EMR for insurance claims or litigation use but are not useful for comprehending a patient's medical history. If all clinical documents in the EMR were automatically disclosed, the browsability of the EHR viewer would decline, and essential medical information might be inadvertently overlooked.

The facilities that can use the proposed EHR system are limited at present to hospitals that have already adopted the DACS. However, it would not be difficult to remodel an EMR to be able to generate a PDF and its ACL file and transfer them to the SS-MIX repository. Furthermore, even with multifunctional network scanners, provided the user registers the document attributes at the time of scanning, the scanned images can be transferred to the SS-MIX repository. For these reasons, our document-based EHR system can be used by welfare facilities that have not yet implemented EMR systems.

\section{Conclusion}

We developed a document-based EHR system through collaboration with the existing EHR system and a documentbased EMR system. The documents are disclosed through the Japanese standard repository SS-MIX, and the document disclosure information is controlled by an ACL file based on the HL7 CDA header. With this system, doctors can disclose not only standardized data but also any clinical documents stored in the document-based EMR system. Furthermore, doctors can select target CDOs and the documents to be disclosed for each patient on a therapeutic department basis.

\section{Acknowledgement}

The document-based EHR system was designed with the collaboration with Fuji Xerox Company. We thank Ryuji Ito, the Director of SEC Corporation, for his contribution toward the realization of our concept.

\section{Author Contribution}

Toshihiro Takeda and Yasushi Matsumura are guarantors of this work, contributed to the proposal of system concept, system design and data analysis. Akito Nakagawa contributed to the system design and determination of the workflow and Shirou Manabe was involved in data extraction and data analysis. Akiko Sakai and Kanayo Ueda contributed in listing and categorization of the medical documents.

\section{References}

[1] Agarwal G, Crooks VA. The nature of informational continuity of care in general practice. Br J Gen Pract. 2008; 58: e17-e24.

[2] Olola CH, Rowan B, Narus S, Hales J, Poynton M, Nebeker J, et al. Enhancing continuity of care through an emergency medical card at Intermountain Healthcare: using Continuity of Care Record (CCR) standard. AMIA Annu Symp Proc. 2007; 11: 1063.

[3] Peters SG, Khan MA. Electronic health records: current and future use. J Comp Eff Res. 2014; 3(5): 515-522.

[4] Delpierre C, Cuzin L, Fillaux J, Alvarez M, Massip P, Lang T. A systematic review of computer-based patient record systems and quality of care: more randomized clinical trials or a broader approach? Int. J. Qual. Health Care. 2004; 16(5): 407-416.

[5] Japanese Association of Healthcare Information System Industry (JAHIS). https://www.jahis.jp/action/ $\mathrm{id}=57$ ? contents_type $=23$

[6] Kimura M, Nakayasu K, Ohshima Y, Fujita N, Nakashima $\mathrm{N}$, Jozaki H, et al. SS-MIX: a ministry project to promote standardized healthcare information exchange, Methods Inf Med. 2011; 50: 131-139.

[7] Matsumoto T, Honda M. The evaluation of the need to share medical data on the community medical ICT network service in Nagasaki, Japan. Stud Health Technol Inform. 2013; 192: 1017.

[8] Campbell SL. HL7 (Health Level 7) - the future becomes a reality. Health Inform. 1990; 7: 24-26.

[9] Health Level Seven ${ }^{\circledast}$ INTERNATIONAL. http://www.hl7. org/implement/standards/product_brief.cfm?product_ $\mathrm{id}=7$

[10] Matsumura Y, Kurabayashi N, Iwasaki T, Sugaya S, Ueda K, Mineno T, et al. A scheme for assuring lifelong readability in computer based medical records. Stud Health Technol Inform. 2010; 160: 91-95.

[11] Chen ES, Melton GB, Engelstad ME, Sarkar IN. Standardizing Clinical Document Names Using the HL7/ 
LOINC Document Ontology and LOINC Codes. AMIA [13] Lee LH, Chou YT, Huang EW, Liou DM. Design of a Annu Symp Proc. 2010; 101-105.

Personal Health Record and Health Knowledge Sharing

[12] IHE Cross Enterprise Document Sharing (XDS) Profile. https://wiki.ihe.net/index.php/Cross-Enterprise Document_Sharing System using IHE-XDS and OWL. J Med Syst. 2013; 37: 9921. 